\title{
Programming Competitions in High School Classrooms: RITA en RED
}

\author{
Vanessa Aybar Rosales \\ LINTI (Laboratory of Research in New \\ Information Technologies) \\ Computer Science School - National \\ University of La Plata \\ La Plata - Buenos Aires - Argentina \\ vaybar@info.unlp.edu.ar
}

Isabel Miyuki Kimura
LINTI (Laboratory of Research in New Information Technologies)
Computer Science School - National University of La Plata
La Plata - Buenos Aires - Argentina ikimura@linti.unlp.edu.ar

\author{
Claudia Queiruga \\ LINTI (Laboratory of Research in New \\ Information Technologies) \\ Computer Science School - National \\ University of La Plata \\ La Plata - Buenos Aires - Argentina \\ claudiaq@info.unlp.edu.ar
}

Matías Brown Bartneche

LINTI (Laboratory of Research in New Information Technologies)

Computer Science School - National University of La Plata

La Plata - Buenos Aires - Argentina mbrown@linti.unlp.edu.ar

\author{
Claudia Banchoff Tzancoff \\ LINTI (Laboratory of Research in New \\ Information Technologies) \\ Computer Science School - National \\ University of La Plata \\ La Plata - Buenos Aires - Argentina \\ cbanchoff@info.unlp.edu.ar
}

\begin{abstract}
This paper presents RITA en RED, an improvement of RITA (Robot Inventor to Teach Algorithms) that came as a results of field tests in high schools of Great La Plata. The goals of the design of RITA en RED are: to promote the introduction of block programming competitions in the classroom, providing a collaborative space for the creation and analysis of programmed robot strategies, while favoring the teaching practice through the development of proposals for teaching programming in the classroom. This paper describes experiences with RITA and the results that led to the design of RITA en RED, preliminary results of a first implementation of RITA en RED and a new design that attempts to improve the experience of the students participating in the competitions.
\end{abstract}

Keywords - Teaching programming, free software, block-based programming, computational thinking, RITA

\section{INTRODUCCIÓN}

En un mundo cada vez más digital, educar en tecnología es imprescindible en la formación de nuestros niños/as y jóvenes. Conocer, entender y operar sobre el mundo que nos rodea, es parte de lo que se espera logren los/as estudiantes en su paso por el sistema educativo. Saber "programar" forma parte de la alfabetización del siglo XXI y debe entenderse como una habilidad técnica en el contexto de la formación de un ciudadano digital que como tal debe ser capaz de entender y modificar su realidad, incluido su entorno digital. Para esto es necesario que las Ciencias de la Computación y en especial la "Programación", se incorpore a los diseños escolares en todos los niveles educativos, como habilitador para el desarrollo de procesos cognitivos vinculados al razonamiento lógico, la descomposición de problemas en partes más simples, la abstracción para manejar la complejidad, la generalización mediante el descubrimiento de patrones y similitudes y la evaluación [1][2][3]. La enseñanza de la programación es hoy un tema de debate en las agendas públicas a nivel global, nuestro país no es ajeno a esto y en los últimos años han surgido iniciativas y políticas federales que sitúan la enseñanza de "programación" en el aula de la escuela como contenido obligatorio [4][5][6]. En el año 2016, en nuestro país, el Ministerio de Educación y Deportes de la Nación lanzó el Plan Nacional Integral de Educación Digital (PLANIED), cuyo objetivo es "integrar la comunidad educativa en la cultura digital, favoreciendo la innovación pedagógica, la calidad educativa y la inclusión socioeducativa. Su misión consiste en que todos los estudiantes de la Argentina adquieran las habilidades necesarias para desenvolverse en el mundo actual y en la sociedad del futuro" [7].

Para la puesta en acción de estos contenidos es necesario disponer de materiales y herramientas cercanas a la cultura de los/as niños/as y adolescentes, que despierten interés y curiosidad por el tema, que contenga un vocabulario que les resulte cotidiano: los juegos de programación, la resolución de desafíos aplicando computación y la robótica educativa son ejemplo de ello. Actualmente existen numerosos proyectos globales y locales enfocados en la enseñanza de la programación, algunos de ellos son las iniciativas globales code.org ${ }^{1}$, codeacademy ${ }^{2} \mathrm{y}$ el proyecto Scratch [8] y, localmente el proyecto PilasBloques ${ }^{3}$. El equipo del LINTI que presenta este artículo trabaja en esta línea de investigación a través de proyectos de investigación y extensión desde el año 2008 [9], siendo RITA (Robot Inventor to Teach Algorithms) uno de los resultados de estas investigaciones [10]. RITA se propone como una herramienta didáctica, cercana a la cultura adolescente que promueve la enseñanza de la programación mediante un abordaje lúdico. Con RITA los/as estudiantes programan juegos de robots virtuales que compiten en un campo de batalla y cuyo desafío es programar una estrategia de robot ganador. El proyecto RITA se inicia como tesina de

\footnotetext{
${ }^{1}$ Sitio oficial de code.org: http://code.org

${ }^{2}$ Sitio oficial de codeacademy: http://www.codecademy.com

${ }^{3}$ Sitio oficial de PilasBloques: http://pilasbloques.program.ar/
} 
grado de Licenciatura en Informática [11] y continúa evolucionando a través de múltiples contribuciones de un equipo de desarrollo conformado en el LINTI. La implementación de RITA en múltiples escuelas secundarias de la localidad del Gran La Plata nos ha permitido evaluar la herramienta, sus potencialidades y debilidades y, a partir de los resultados obtenidos surge la propuesta de RITA en RED, como herramienta superadora a RITA.

Este artículo presenta RITA en RED, cuyo diseño ha sido guiado por dos objetivos estrechamente relacionados:

- Promover el desarrollo de competencias de programación en bloques en el aula de la escuela, brindando un espacio colaborativo de creación y de análisis de las estrategias de robots programadas.

- Favorecer la tarea docente a través del desarrollo de propuestas áulicas en torno a la enseñanza de programación.

Este artículo comienza describiendo las experiencias del trabajo con RITA como insumo para la propuesta de RITA en RED. Luego se presenta el primer diseño e implementación de RITA en RED junto con los resultados y desafíos que surgen de la puesta en acción. Finalmente se presenta un nuevo diseño de RITA en RED, los resultados preliminares y se delinean los trabajos futuros.

\section{RITA: UNA HERRAMIENTA DIDÁCTICA PARA ENSEÑAR A PROGRAMAR}

RITA es una herramienta didáctica, cercana a la cultura adolescente que promueve la enseñanza de programación mediante un abordaje lúdico. Con RITA los estudiantes programan con bloques las estrategias de combate de robots virtuales que compiten en un campo de batalla y cuyo desafío es programar estrategias de robots ganadores. Las batallas de robots en RITA no promueven la violencia, no involucran personas, no contienen sangre ni plantean situaciones de enfrentamiento racial, el objetivo de las batallas de RITA es promover la competencia de estrategias, programas, en un sentido positivo.

RITA es una aplicación JAVA de código fuente abierto que extiende, integra y adapta las funcionalidades de dos frameworks de código fuente abierto, Openblocks ${ }^{4}$ y Robocode $^{5}$. Openblocks [12] brinda el soporte para la programación con bloques gráficos que recrean el uso de las piezas de LEGO ${ }^{6}$. En RITA el framework Openblocks fue extendido para brindar soporte a las clases JAVA provistas por el framework Robocode, así como también proveer las estructuras de control, funciones y operadores de JAVA.

Los bloques de RITA representan acciones que el robot realiza en el campo de batalla, varias de estas acciones son para desplazarse en el campo de batalla y en respuesta a eventos que ocurren en su entorno, por ejemplo cuando se choca contra un muro, cuando otro robot lo ataca, entre otros. Asimismo, RITA

\footnotetext{
${ }^{4}$ OpenBlocks: http://education.mit.edu/?s=openblocks

${ }^{5}$ Robocode: http://robocode.sourceforge.net/

${ }^{6}$ LEGO: https://www.lego.com/es-ar/
}

cuenta con un amplio conjunto de bloques para realizar operaciones aritméticas, trigonométricas, lógicas, para manipular variables, etc. agrupadas bajo categorías de bloques. La Fig. 1 muestra el ambiente de trabajo de RITA: el panel de la izquierda contiene las categorías que agrupan a los bloques disponibles, el panel central es el área de trabajo principal en el que se programa la estrategia del robot; asimismo en este panel está disponible la ventana que muestra el código JAVA, que RITA automáticamente traduce, y que puede habilitarse/deshabilitarse a demanda. Este elemento puede usarse como puente para unir el código programado con bloques con el código escrito en un lenguaje de programación textual.

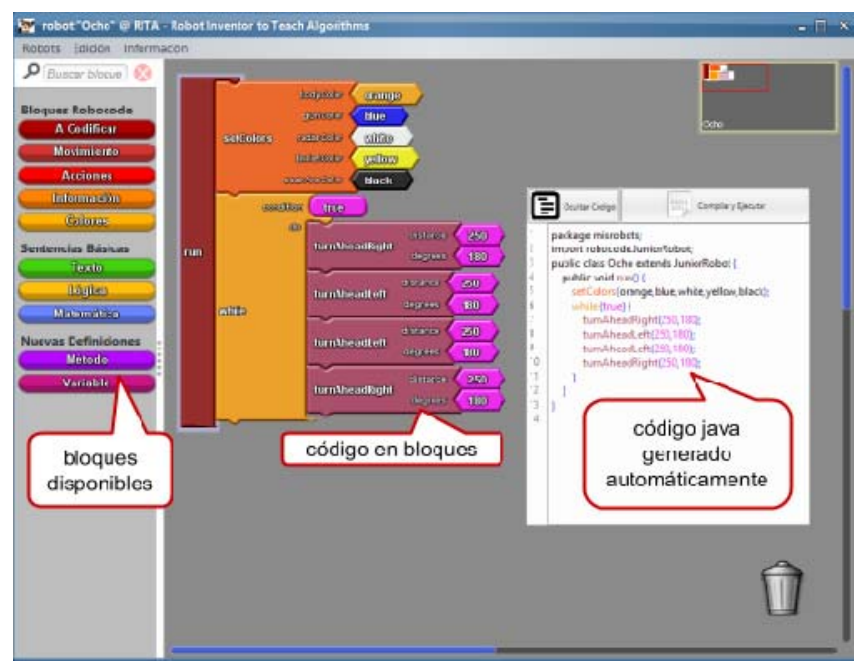

Fig. 1. Ambiente de programación de RITA. Los bloques disponibles del panel de la izquierda pueden arrastrarse al área central donde se programa la estrategia del robot.

Robocode [13] brinda un conjunto de clases JAVA que representan a los robots y la interacción entre ellos, el soporte para la ejecución de los robots y el motor de ejecución de la batalla. Robocode es un juego de programación cuyo objetivo es programar en JAVA la estrategia de un robot para competir con otros robots en un campo de batalla. El jugador es el programador del robot y mediante el código que escribe le brinda inteligencia al robot, indicando cómo comportarse y reaccionar frente a eventos ocurridos en el juego. Robocode provee a RITA de un campo de batalla, donde pequeños robots virtuales compiten. La Fig. 2 muestra una pantalla de RITA con robots compitiendo en el campo de batalla de Robocode.

RITA provee un entorno de programación integrado en el que los/as estudiantes "programan con bloques" las estrategias de combate de sus robots y luego las prueban en el campo de batalla de Robocode. En esta experiencia de juego con RITA se exploran conceptos de programación tales como: secuencia, iteración, eventos, paralelismo, estructuras de control, operadores matemáticos, expresiones lógicas y variables, que se ponen en acción en la resolución de problemas aplicando un enfoque incremental e interactivo, en el que se testean y corrigen errores a medida que se desarrolla la solución. 


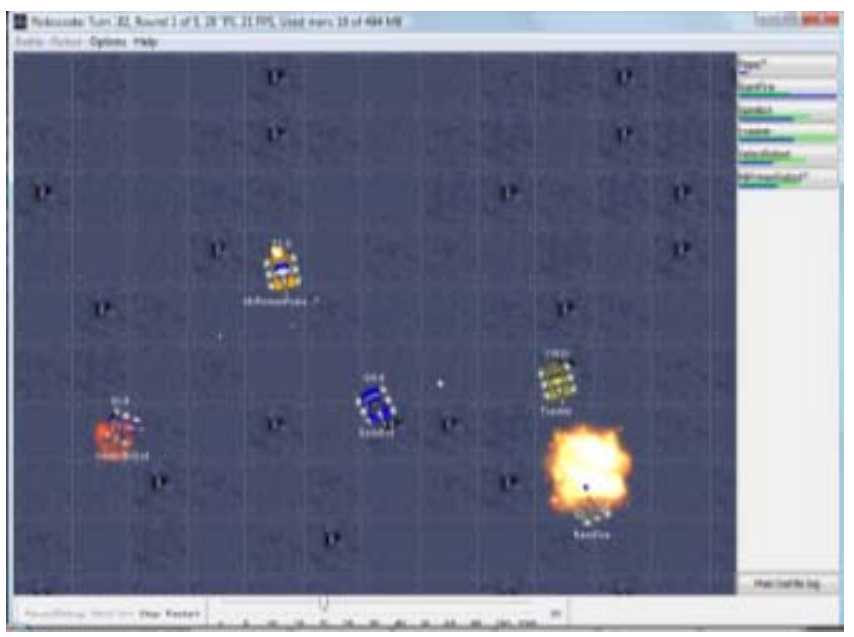

Fig. 2. Campo de batalla de Robocode. En el margen derecho se muestra información de los robots participantes y su progreso en el juego.

RITA es una herramienta libre, de código fuente abierto, disponible para su descarga desde https://github.com/vaybar/RITA.

\section{A. Las experiencias con RITA}

Las experiencias con RITA comenzaron en el año 2012 a partir del proyecto de extensión "Articular universidad-escuela con JAVA para fortalecer la Educación-Técnica" [14]. Se continuó a través de proyectos de investigación y extensión de la UNLP en los que se prioriza el trabajo de campo en escuelas de gestión pública. En el desarrollo de las experiencias con RITA se pueden identificar diferentes fases:

\section{1) Fase 1}

En esta fase inicial se trabajó con docentes y estudiantes de cuatro escuelas secundarias técnicas de la Provincia de Buenos Aires con orientación informática. Se incorporó a modo de experiencia piloto RITA, como herramienta para favorecer el trabajo con contenidos de programación en los espacios curriculares propios de la orientación. RITA fue primero probada con los docentes y una vez evaluada favorablemente como material didáctico acorde a las necesidades de sus espacios curriculares, se organizaron talleres con los estudiantes. La prueba piloto se organizó en dos etapas: la primera con un grupo reducido de estudiantes de las cuatro escuelas participantes del proyecto de extensión y posteriormente se amplió la prueba a cursos completos de las cuatro escuelas. A partir de esta primera fase se inicia la preparación del material de acompañamiento a los docentes sobre programación de juegos de robots virtuales con RITA. Participaron de la experiencia 10 docentes y 116 estudiantes de escuelas técnicas con orientación informática.

\section{2) Fase 2}

Se continúa trabajando con las mismas escuelas y se incorporan escuelas secundarias no-técnicas en algunas actividades. Se focaliza el trabajo en el material didáctico de acompañamiento a los/as docentes, en la incorporación de mejoras a RITA, en el diseño de actividades nuevas para los/as estudiantes y en la actualización de cuestiones didácticas y visuales del material, de modo de hacerlo más atractivo para los/as estudiantes. Se constituyó un grupo de trabajo formado por docentes de las escuelas y el equipo de desarrollo de RITA con la meta de diseñar y planificar las actividades destinadas a los/as estudiantes, que permitan recorrer la temática de los contenidos de programación en forma gradual.

Durante esta fase del proyecto surgen, por parte de los docentes, propuestas de mejoras para RITA con el objetivo que los/as estudiantes puedan programar robots que no compitan con otros robots, simplemente que puedan hacer pruebas simples en las que no consideren a otros robots. Por ejemplo, permitir que se programen robots que dibujen figuras geométricas imaginarias, sin trazo, en el plano. El sentido de estas incorporaciones está dado porque la formación de los estudiantes de la escuela secundaria no-técnica no contempla contenidos de programación, entonces fue necesario adaptar tanto RITA como el material para facilitar el acercamiento a la programación. Este tipo de actividades son más sencillas y proveen un acercamiento más simple a los primeros conceptos de programación. Participaron en estas experiencias 60 estudiantes.

\section{3) Fase 3}

En esta fase se comenzó a trabajar en el espacio curricular Nuevas Tecnologías de la Información y la Comunicación (NTIC) de una escuela secundaria del Gran La Plata con dos cursos de 4to año de la escuela. La experiencia fue muy positiva y valorada por la comunidad de la escuela. Esto nos permitió participar en el evento Universo PROGRAM.AR realizado en Tecnópolis en octubre de 2014 (http://goo.gl/H08LV3) .

Se continúa trabajando en la mejora del material. De estas experiencias participaron 53 estudiantes de 4to año.

\section{4) Fase 4}

En esta etapa se avanzó en el material de acompañamiento para docentes, se realizaron talleres de los que participaron estudiantes y docentes de escuelas secundarias técnicas y notécnicas de la localidad del Gran La Plata y se amplía la propuesta a las escuelas preuniversitarias de la UNLP.

En esta fase del proyecto se comienza a trabajar en la primera versión de RITA en RED. Esta nueva propuesta permitirá mejorar la experiencia de uso de docentes y estudiantes, facilitando la realización de las competencias en forma automática, fortaleciendo el rol de mediador del docente y el trabajo en equipos.

\section{B. Metodología de trabajo con RITA}

El trabajo en las aulas está impulsado por los/as docentes que, en primer lugar se capacitan en RITA y luego comienzan a pensar cómo incorporar los contenidos a sus espacios curriculares.

La metodología de trabajo en el aula es de taller, se utilizan las computadoras disponibles en la escuela y los estudiantes trabajan en equipos. Los talleres se estructuran en cuatro encuentros bajo el lema "Programar juegos de robots con RITA". Las actividades de los encuentros son los siguientes:

\section{1) Primer encuentro: Festival de instalación de RITA}


En este primer encuentro se propone a los estudiantes que instalen RITA en sus computadoras ${ }^{7}$ con el seguimiento y asistencia de los/as docentes. En las escuelas que disponen de una Intranet, los/as estudiantes descargan RITA de la red.

Seguidamente se propone a los/as estudiantes que exploren RITA y que realicen actividades específicas que les permita reconocer los principales bloques y su funcionamiento.

2) Segundo encuentro: Entrenamiento en la construcción de juegos de robots con RITA-Primera Parte

Se trabajan conceptos de programación proponiendo la resolución de desafíos sencillos: desplazamiento de los robots en el campo de batalla de RITA aplicando cálculos aritméticos $\mathrm{y}$ trigonométricos simples. Se realizan las primeras competencias con robots preinstalados en RITA.

3) Tercer encuentro: Entrenamiento en la construcción de juegos de robots con RITA-Segunda Parte

Se continúa trabajando conceptos de programación y se incorpora la noción de eventos, proponiendo la resolución de desafíos más complejos que involucran cuestiones como la administración de la energía los robots y el uso del radar para detectar otros robots en el campo de batalla, etc. Se propone a los equipos: pensar en estrategias defensivas y ofensivas para sus robots, para ello se realizan competencias a modo de prueba para testear el funcionamiento del robot programado por los equipos y, evaluar y analizar el funcionamiento de las estrategias programadas haciendo los ajustes necesarios teniendo en cuenta que el robot participará de una competencia con los robots de los/as compañeros/as del aula.

\section{4) Cuarto encuentro: Competencias de robots con RITA}

En este último encuentro se realiza una competencia de robots en el aula de la que participan todos los robots programados en un campo de batalla común y de la que sólo un robot resultará el ganador.

Se organiza la competencia: cada equipo deberá programar una estrategia de robot personalizada que combine estrategias ofensivas y defensivas, se propone que prueben las estrategias y se asigna un tiempo para la programación del robot.

Para la puesta en acción de la competencia es necesario colectar todas las estrategias programadas en una computadora. RITA no provee de un mecanismo de comunicación que permita "enviar" un robot a otra computadora, por ello los robots deben "copiarse" manualmente usando algún dispositivo de almacenamiento removible como por ejemplo un pendrive, de modo de centralizarlos en una máquina seleccionada para que ejecute la competencia de las estrategias de los robots. Una vez copiados todos los robots, comienza la competencia que finaliza cuando sólo un robot resulta ganador.

Este encuentro tiene sumo interés didáctico: promueve el trabajo en equipos, la colaboración y estimula las habilidades en la resolución de problemas aplicando computación.

\footnotetext{
${ }^{7}$ En todas las escuelas participantes, los estudiantes poseen las netbooks entregadas por el Programa Conectar-Igualdad.
}

\section{Evaluación}

A lo largo de las diferentes experiencias realizadas, los docentes destacaron que la propuesta de trabajo con RITA les permite acercar los contenidos de programación de una manera sencilla y motivadora, dado que a los/as estudiantes les resulta próximo el formato de juego y las pantallas forman parte de su escena más cotidiana.

Se administraron encuestas a los/as estudiantes en las diferentes fases de las experiencias con RITA cuyos resultados evidenciaron que RITA es una herramienta interesante para introducir en el aula de escuela secundaria en los espacios curriculares propios de programación o NTIC.

De las experiencias de trabajo de campo se relevaron inquietudes y sugerencias por parte de docentes y estudiantes, las cuales se incorporaron como mejoras en las versiones de RITA. Una propuesta que surgió desde el propio equipo de desarrollo de RITA y de las experiencias realizadas fue mejorar la interacción entre los equipos de estudiantes para intercambiar las estrategias de sus robots utilizando la conectividad de red disponible en la escuela. De este nuevo módulo se espera que sea simple de configurar por los/as estudiantes, considerando que si bien todos son usuarios de redes e Internet, no necesariamente conocen y manejan los conceptos de conectividad.

\section{RITA EN RED: LA PRIMERA APROXIMACIÓN}

RITA en RED surge como una mejora de RITA en relación a experiencia de uso en las competencias de programación de robots. Con el objetivo de evaluar que efectivamente la incorporación de la comunicación en red mejora la experiencia en el uso de RITA, surge la propuesta de desarrollar una primera versión a modo experimental de RITA en RED.

\section{A. La primera versión de RITA en RED}

Esta primera aproximación sigue los lineamientos de la arquitectura cliente-servidor. La solución consiste en delegar en el servidor la recepción de las estrategias a competir (código fuente) y la generación de un archivo binario que contiene el "paso a paso" de la ejecución de la competencia de los robots. La Fig. 3 muestra la arquitectura de la primera versión de RITA en RED.

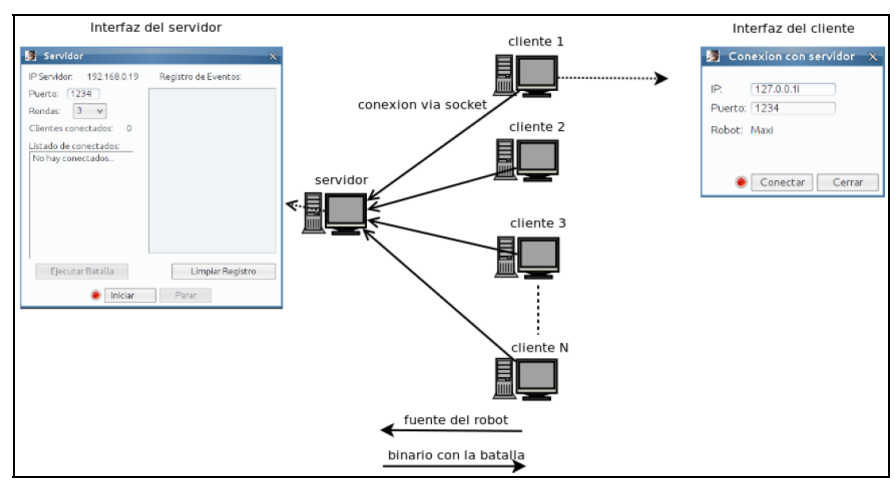

Fig. 3. Arquitectura de RITA en RED, primera versión 
La interfaz gráfica de RITA fue modificada para permitir configurar los parámetros de la conexión que permiten a cada equipo conectarse a la red: a) en el servidor se configura el número de puerto donde se escuchan las conexiones y el número de rondas de la competencia; también se puede visualizar la cantidad de clientes conectados y los nombres de los mismos (la Fig. 4 muestra la ventana de configuración del servidor) y b) los clientes se conectan con el servidor para enviar el código fuente de la estrategia de un robot, así como también para recibir el resultado de la ejecución de la competencia (archivo binario generado por el servidor). En el cliente se realizan configuraciones para establecer la conexión con el servidor: dirección IP y puerto donde está escuchando el servidor por pedidos de conexión.

Todas las opciones de configuración están integradas en RITA en RED a través de una nueva opción del menú superior que permite elegir si el equipo funciona como servidor o como cliente, y además una nueva opción sobre el panel que muestra el código fuente de la estrategia llamada: "Jugar en RED", mostrado en la Fig. 5.

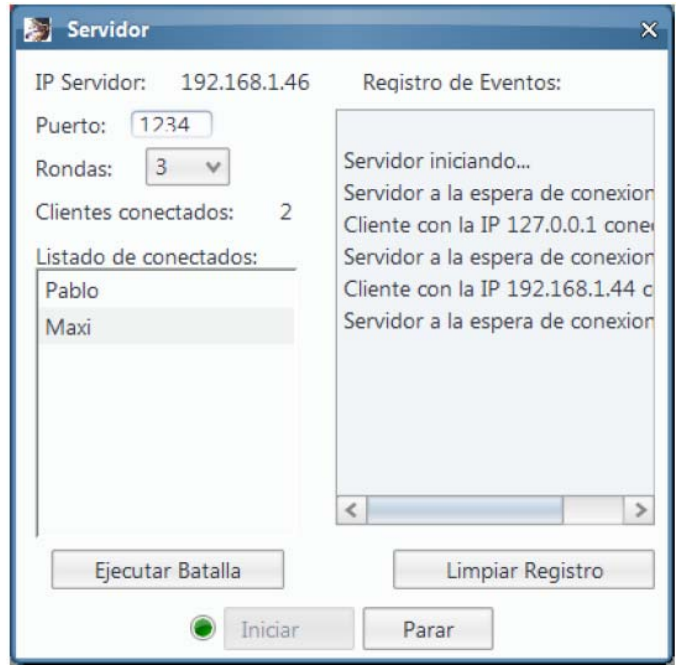

Fig. 4. Ventana de configuración del servidor de RITA en RED

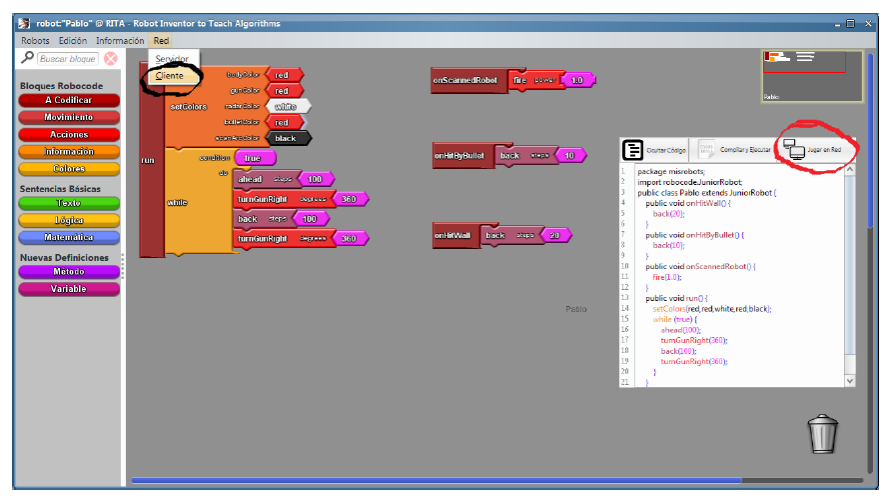

Fig. 5. Interfaz gráfica de RITA en RED

A nivel de implementación, los clientes y el servidor establecen conexión haciendo uso de la API de networking de Java, vía Sockets TCP, orientada a conexión brindando seguridad y estabilidad en la comunicación. En RITA en RED cada conexión entre el servidor y un cliente es atendida por un hilo de ejecución debido al carácter sincrónico del tipo de conexión. A través de los mensajes enviados entre el cliente y el servidor, se va llevando adelante en forma secuencial el envío y recepción de los datos necesarios para realizar la tarea, tanto mensajes de sincronización como los archivos que representan el código fuente de los robots y el archivo binario con la resolución de la batalla.

\section{B. Experiencia en el aula}

La primera evaluación de RITA en RED se realizó mediante una prueba de campo en una de las escuelas secundarias con las que habitualmente se trabaja con RITA, con estudiantes de cuarto y quinto año. Participaron de la experiencia 24 estudiantes que utilizaron RITA en experiencias previas, familiarizados con la herramienta, y un docente de la escuela. En esta prueba de campo se usaron las netbooks de los estudiantes y se organizó en 2 momentos:

\section{1) Organización de la competencia grupal}

En este momento se organizó la competencia en modalidad de finales parciales: cada equipo formado por dos estudiantes programan una estrategia que luego compite en una final en la que participan cuatro grupos. Los equipos ganadores compiten en una semifinal hasta llegar a una final de la que saldrá un único equipo ganador. Se acordó hacer las competencias en 3 rondas.

El equipo de desarrollo RITA en RED configuró tres redes diferentes y asignó a cada equipo una red para las competencias. Los estudiantes eligieron una de las netbooks como equipo servidor.

\section{2) Competencia grupal}

Se ponen en común algunas ideas sobre las estrategias defensivas y ofensivas y se propone a los estudiantes que desarrollen su estrategia en un tiempo establecido.

Los equipos se unen a la red asignada, una vez desarrollada la estrategia, para competir con cuatro equipos; los ganadores compiten en semifinales hasta llegar a la final. En esta competencia ganó el robot llamado "Desapitodominaargentina".

Además a modo de comprobar la inteligencia de los robots en forma independiente de los grupos, se propuso realizar una única competencia grupal de la que participan todos los robots. Todas las netbooks se unieron a una misma red. De esta competencia también resultó ganador "Desapitodominaargentina".

\section{Evaluación}

Se evaluó mediante una encuesta la experiencia de los estudiantes con esta nueva versión de RITA. Se buscó indagar sobre diferentes aspectos:

- la complejidad en el uso de RITA en RED, teniendo en cuenta que los/as estudiantes en general desconocen conceptos como cliente, servidor, dirección IP, puerto, etc., 
- la eficiencia en relación al tiempo de respuesta para obtener el resultado de la competencia una vez enviado el robot,

- la motivación de poder competir en red en contraposición a las competencias "manuales" y,

- cómo calificarían la experiencia y qué mejoras incorporarían.

Los resultados de la encuesta reflejan distintas conclusiones:

- La complejidad de RITA en RED: para el 82,4\% resultó de complejidad media, para el $17,6 \%$ resultó fácil y ninguno de los/as estudiantes calificó como difícil o imposible. Un 47,1\% manifestó entender los conceptos de cliente, servidor, dirección IP y puerto, mientras que un 35,3\% manifestó entenderlo a medias, y el 17,6\% no los comprendieron.

- El tiempo de respuesta: para el 17,6\% de los/as estudiantes fue óptimo, para el $41.2 \%$ fue bueno y para el $35.3 \%$ fue lento, un $5,9 \%$ no respondió la pregunta relacionada a este tema.

- La motivación de competir en una red: al 94\% de los/as estudiantes les resultó más cómodo enviar el robot a competir usando la nueva funcionalidad de "Jugar en RED" que utilizar un pendrive. Asimismo el $52.9 \%$ de los/as estudiantes respondió que los motiva y entusiasma programar en un juego en red, mientras que al $35.3 \%$ le entusiasma poco y un $11.8 \%$ no lo entusiasma. El $52.9 \%$ de los/as estudiantes manifestaron que competirán con amigos o compañeros fuera del aula $\mathrm{y}$ un $41.2 \%$ posiblemente lo haga, mientras que un $5.9 \%$ no contestó la pregunta relacionada al uso fuera de la escuela.

- La calificación de la experiencia y mejoras que incorporarían: esta pregunta fue libre y se recolectaron varias opiniones:

- un gran porcentaje opinó que RITA tendría que contar con la opción de idioma "castellano"

○ un gran porcentaje opinó que "está bueno y es divertido" programar con RITA en RED por la posibilidad de poder jugar y competir con sus compañeros en batallas de robots.

- algunos estudiantes opinaron que les gustaría poder personalizar visualmente sus robots.

De los resultados de las encuestas podemos concluir en términos generales que RITA en RED tuvo buena aceptación por parte de los/as estudiantes. La experiencia en la escuela resultó positiva y permitió evaluar esta nueva modalidad de trabajo en el aula.

La versión de RITA en RED con una arquitectura cliente/servidor cumple con el objetivo de simplificar la comunicación entre los equipos participantes de las competencias.
La evaluación del equipo de desarrollo de RITA, luego de haber observado la experiencia áulica, consideró que es necesario trabajar en una nueva versión de RITA en RED que rescate las ventajas de jugar en red y elimine las siguientes cuestiones:

- Configuración cliente/servidor: implica introducir a los/as estudiantes en conceptos de redes, explicar cuestiones acerca de la configuración que están realizando: ¿qué significa ser cliente? y ¿qué significa ser servidor?, ¿qué es una IP?, ¿qué es un número de puerto?, si quiero hacer funcionar mi netbook como servidor ¿cómo sé cuál es su IP?. Estas preguntas desplazan el foco de la enseñanza de programación. La pregunta sobre este tema pone en evidencia que para la mitad de los estudiantes resultó complejo entender la configuración de RITA en RED.

- El trabajo del servidor: el servidor resuelve la competencia y está en condiciones de visualizar el resultado, sin embargo las netbooks cliente no pueden hacerlo hasta que reciben la competencia. En realidad no hay concurrencia, es una competencia en diferido. Para ejemplificar: el servidor debe "grabar un video" de la competencia y luego lo envía al resto de las netbooks del grupo para que lo reproduzcan.

Un punto a favor en este tipo de resolución es que con certeza todos los miembros del grupo visualizarán exactamente el mismo resultado. Como contrapartida, hay que tener en cuenta que el desarrollo de una competencia de estrategias -el paso a paso- puede ocupar varios MB dependiendo de su duración y ésta es la información que debe ser enviada por la red a cada uno de los integrantes. En este punto estamos dependiendo del hardware (router) con el cual se disponga en el aula de clase, la latencia en el arribo de la información a su destino hará que las visualizaciones de las batallas en las distintas netbooks sea a destiempo. Es decir, no está garantizado que todos/as los/as estudiantes vean el resultado al mismo tiempo. En términos de competencia se pierde el interés por visualizar el resultado si ya lo conozco.

- Disponibilidad de routers: considerando que la cantidad de información a intercambiar entre las netbooks puede resultar de varios MB, es recomendable contar con routers que distribuyan el tráfico y así se logre un tiempo de respuesta razonable.

\section{RITA EN RED: UN NUEVO DISEÑO}

A partir de la evaluación de primera versión de RITA en RED se diseñó una nueva versión bajo los siguientes lineamientos:

- Brindar la posibilidad de establecer grupos de competencia de manera transparente, de modo de evitar hacer configuraciones que resulten distractoras.

- Mejorar el uso de la red, intercambiando sólo las estrategias de los robots del grupo, y no la competencia completa. De esta forma la estrategia ganadora se 
debería resolver en cada una de las netbooks. Y aquí un punto no menor es que debe asegurarse que el desarrollo y el resultado es exactamente el mismo en todas las netbooks.

- Resolver el problema del determinismo relacionado al punto anterior. En RITA se pueden usar valores aleatorios para programar las estrategias, por tanto en un principio el resultado de la competencia de las estrategias no necesariamente terminará en el mismo resultado.

\section{A. El mecanismo de comunicación}

Este diseño propone el uso de una comunicación multicast, distribuida. Se probaron y evaluaron diferentes implementaciones de multicast en JAVA y se decidió utilizar $\mathrm{JGroups}^{8}$ por su carácter de código fuente abierto, por proveer confiabilidad en el intercambio de mensajes y por la transparencia en el manejo de direcciones IP.

Las computadoras que participan de un grupo que competirá tienen un nombre lógico que es administrado internamente por JGroups, de este modo cuando un estudiante desea unirse a un grupo, simplemente elegirá un número grupo. El/la estudiante no necesita conocer cómo funciona internamente el grupo ni usar una dirección IP en particular, ni actualizar la información de su robot de manera explícita ante cualquier cambio en la estrategia programada, sino que es el software de comunicación el que se encarga de administrar la comunicación entre los miembros del grupo, de un modo transparente El estudiante solo debe indicar a qué grupo quiere unirse y cuándo lo abandona.

La Fig. 6 muestra la nueva arquitectura de comunicación de RITA en RED: a diferencia de la arquitectura de la versión anterior, no hay clientes y servidor, sino que cada estudiante conectado con una computadora enviará su robot al resto de los estudiantes pertenecientes al mismo grupo que participa de la competencia. En esta nueva arquitectura el tiempo consumido en intercambio de información se reduce notablemente dado que sólo se envía la estrategia del robot que competirá y la batalla se resuelve en forma distribuida en cada una de las computadoras que participan, mientras que en la arquitectura anterior la información a intercambiar ocupa varios MB, dado que se envía la ejecución de la batalla resuelta.

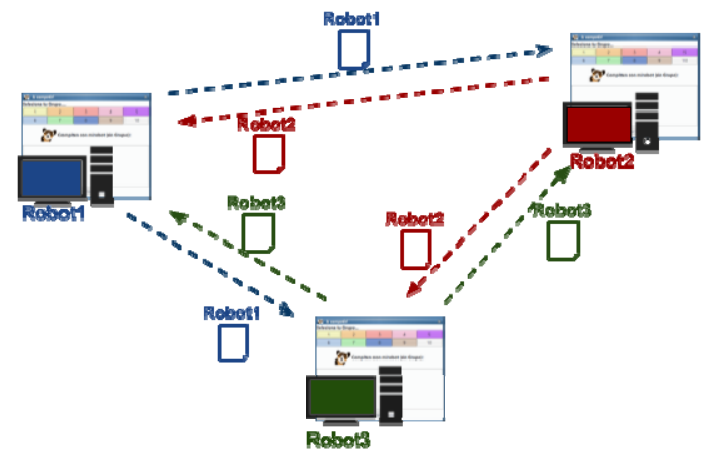

Fig. 6. Arquitectura de RITA en RED, versión mejorada

\footnotetext{
${ }^{8}$ JGroups: http://jgroups.org/
}

\section{B. Las competencias y el problema del determinismo}

La nueva solución plantea la ejecución de la competencia de modo descentralizado. Para que la batalla sea justa, las posiciones iniciales de los robots participantes deben ser aleatorias. El problema aquí planteado es cómo asegurar que todas las posiciones iniciales elegidas por los participantes sean las mismas en las diferentes computadoras, y todo cálculo aleatorio dé el mismo resultado, de modo que la resolución de la competencia sea la misma en todas las computadoras participantes.

RITA en RED brinda dos modos de ejecución de las estrategias programadas, la Fig. 7 muestra la pantalla que permite acceder a estos dos modos de ejecución.

- Modo "Entrenamiento": este modo es similar a la funcionalidad existente en RITA, cada equipo de estudiantes puede individualmente "Probar" su estrategia seleccionando otros robots preinstalados, para medir su desempeño. Los valores empleados en las estrategias programadas son efectivamente aleatorios en función de las capacidades del lenguaje.

- Modo "Competencia": a este modo se accede mediante la opción "Competir". Esta opción le permite al estudiante seleccionar el grupo en donde compite, o, si seleccionó un grupo, iniciar la competencia contra los miembros del grupo seleccionado. En modo "Competencia", la generación de números aleatorios se establece sobre un mismo valor inicial en todas computadoras que participan de la competencia y el framework Robocode se configura de modo que se garantice la elección determinística de los valores.

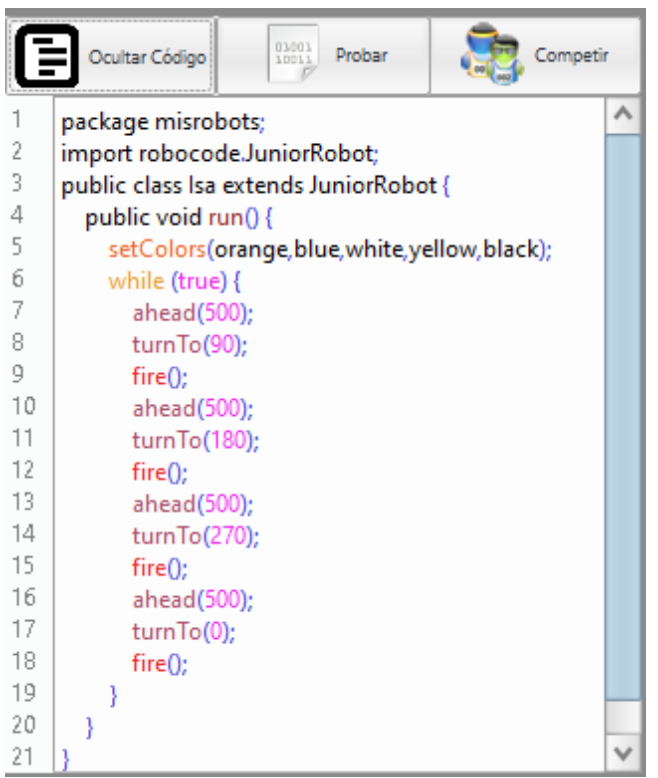

Fig. 7. Interface de la ventana ejecutar las estrategias: "Probar" y "Competir".

\section{Evaluación}

Los primeros testeos de esta nueva versión de RITA en RED se realizaron en el LINTI con cinco computadoras. Se pudo probar el intercambiando sólo de robots entre las 
computadoras y realizar la competencia en forma distribuida en cada uno de los equipos del grupo. El resultado de la competencia en las distintas netbooks fue el mismo, siendo ésto muy alentador en el sentido que el problema de la falta de determinismo pudo resolverse. Asimismo el tiempo de respuesta mejora notablemente respecto de la primera versión de RITA en RED en la que el intercambio resultaba costoso en términos de byte transferidos.

Asimismo de estas evaluaciones surgieron propuestas de cambios en la interfaz de usuario que permitirán mejorar la experiencia de los/as estudiantes que participan en las competencias. A modo de ejemplo, luego de una competencia un equipo de estudiantes podría obtener el programa en bloques (código fuente) del robot de un competidor, previa autorización, de modo de entender la estrategia del oponente e incluso mejorarla. También se podrían incorporar características de gamification, para premiar no sólo al ganador de una batalla sino a la práctica en el modo "Entrenamiento".

\section{CONCLUSIONES}

Los objetivos principales de la enseñanza de programación en los sistemas educativos es promover el desarrollo de habilidades técnicas vinculadas a la capacidad de resolver problemas usando computación, a la creatividad, a la colaboración, el pensamiento crítico y a la comunicación. Estos procesos deben ser acompañados con herramientas y materiales próximos a los/as niños/as y adolescentes y que puedan implementarse con los recursos tecnológicos disponibles en las escuelas.

El proceso de desarrollo de RITA en RED nos permite obtener algunos resultados preliminares:

- Los/as estudiantes se manifiestan altamente motivados por las competencias en red tanto en el aula como en otros espacios comprometiéndose en su propio aprendizaje.

- Los docentes manifestaron que la experiencia en la escuela resultó positiva y les permite evaluar esta nueva modalidad de trabajo en el aula.

- Se encontraron elementos distractores en la primera versión de RITA en RED vinculados al manejo de redes que fueron eliminados en el siguiente diseño.

- El tiempo de respuesta de la primera versión de RITA en RED no fue el esperado por los/as estudiantes ni por el equipo de desarrollo RITA, requiriendo revisar y adaptar la arquitectura de comunicación. Sin embargo, disponer de una versión para hacer una prueba de conceptos fue altamente valorada por el equipo.

Actualmente nos encontramos en una etapa de prueba y depuración de una nueva versión desarrollada bajo una arquitectura de comunicación distribuida, que optimiza el tiempo de respuesta en el intercambio de estrategias de robots y que incluirá algunas características de gamification que abonarán a mejorar la experiencia de uso de RITA en RED.

\section{AGRADECIMIENTOS}

A los estudiantes de Licenciatura en Sistemas de la Facultad de Informática de la UNLP, Pablo Vilaltella y Maximiliano Rugna, por su aporte en el desarrollo de la primera versión de RITA en RED.

\section{REFERENCIAS}

[1] C. Queiruga, C. Banchoff Tzancoff, S. Martín, V. Aybar Rosales, F. López, I. Kimura y N. Gómez, "PROGRAMAR en la Escuela: nuevos desafíos en las aulas", presentado en la $19^{\circ}$ Edición del Workshop de Investigadores en Ciencias de la Computación, Buenos Aires, Argentina, Abril 27-28, 2017. En proceso de publicación.

[2] F. Tarasow, "Enseñar a codificar, ¿la nueva alfabetización digital?", columna del coordinador del PENT - FLACSO, Marzo 7, 2017. [Online]. Disponible: http://www.pent.org.ar/fabiotarasow/ensenarcodificar-nueva-alfabetizacion-digital

[3] K. Brennan y M. Resnick, "New frameworks for studying and assessing the development of computational thinking", Proceedings of the 2012 annual meeting of the American Educational Research Association, Vancouver, Canada, April 2012.

[4] National curriculum in England: computing programmes of study. [Online]. Disponible: https:/www.gov.uk/government/publications/national-curriculum-inengland-computing-programmes-of-study, 2013.

[5] Resolución 263/15. Ministerio de Educación de la Nación Argentina. [Online]. Disponible: http://www.me.gov.ar/consejo/resoluciones/res15/263-15.pdf.

[6] Program.AR: programa de la Fundación Sadosky, Ministerio de Ciencia, Tecnología e Innovación Productiva de la Nación Argentina. [Online]. Disponible: http://www.fundacionsadosky.org.ar/programas/programar/

[7] Plan Integral de Educación Digital del Ministerio de Educación y Deportes de la Nación Argentina [Online]. Disponible: http://planied.educ.ar/category/novedades/

[8] M. Resnick et al., "Scratch: Programming for All", Communications of the ACM, vol. 52, no. 11, pp. 60-67, November 2009, doi:10.1145/1592761.1592779

[9] J. Díaz, C. Banchoff Tzancoff, C. Queiruga, E. S. Martin. "Experiencias de la Facultad de Informática en la Enseñanza de Programación en Escuelas con Software Libre", Memorias del Congreso Iberoamericano de Ciencia, Tecnología, Innovación y Educación 2014, Buenos Aires, Argentina, Noviembre 12-14. ISBN 978-84-7666-210-6. Artículo 1426. [Online]. Disponible: http://www.oei.es/congreso2014/memoriactei/1426.pdf

[10] V. Aybar Rosales, C. Queiruga, I. Kimura, M. Brown Barnetche, N. Gómez. "Enseñando a programar con RITA en escuelas secundarias", Proceedings del XXI Congreso Argentino de Ciencias de la Computación (CACIC 2015), Junín, Argentina, Octubre 5-9, 2015. ISBN 978-987-3724-37-4, pp. 1201-1211. [Online]. Disponible: http://sedici.unlp.edu.ar/handle/10915/50644.

[11] V. Aybar Rosales, "Aplicaciones complementarias a Robocode que faciliten el aprendizaje de programación en escuelas secundarias". Tesis de Licenciatura en Informática, Facultad de Informática, UNLP, Argentina, 2012. [Online]. Disponible: http://sedici.unlp.edu.ar/handle/10915/47050.

[12] [R. Roque, "OpenBlocks: An extendable framework for graphical block programming systems". M. S. Thesis. Massachusetts Institute of Technology, Cambridge, MA, 2007.

[13] M. Nelson, "Robocode: Build the best - destroy the rest!" [Online]. Disponible: http://robocode.sourceforge.net/.

[14] C. Queiruga, L. Fava, "Articular Universidad Escuela: una experiencia de intervención de la Facultad de Informática de la UNLP ", Anales del $10^{\circ}$ Simposio sobre la Sociedad de la Información (JAIIO-SSI), La Plata, Argentina, Agosto 2012. ISSN-L: 1666-1141. 